\title{
NUEVO REGISTRO DE APHAENOGASTER PUNCTATICEPS MACKAY, 1989 PARA MÉXICO Y PRIMER REGISTRO DE APHAENOGASTER MEXICANA (PERGANDE, 1896) PARA JALISCO (HYMENOPTERA: FORMICIDAE: MYRMICINAE)
}

Villalvazo-Palacios, M., Pérez-Domínguez, J. F. \& Vásquez-Bolaños, M. 2014. New record of Aphaenogaster punctaticeps Mackay, 1989 for Mexico and first record of Aphaenogaster mexicana (Pergande, 1896) for Jalisco state (Hymenoptera: Formicidae: Myrmicinae). Acta Zoológica Mexicana (n. s.), 30(2): 431-433.

ABSTRACT. We present a new record of Aphaenogaster punctaticeps Mackay, 1989 from Mexico and the first record of Aphaenogaster mexicana (Pergande, 1896) from Jalisco State. The specimens were collected in Sierra de Quila, Jalisco, Mexico. With the new records now, there are 13 species of the genus Aphaenogaster from Mexico and three from Jalisco: Aphaenogaster ensifera Forel, 1899, A. mexicana (Pergande, 1896) and A. punctaticeps (Mackay, 1989).

El género Aphaenogaster pertenece a la tribu Pheidolini, de la subfamilia Myrmicinae. Presenta el escapo largo que va más allá del borde posterior de la cabeza, sin maza antenal; cabeza alargada y angosta hacia el vertex formando un cuello posterior; la mandíbula es delgada y triangular; son hormigas monomórficas o muy levemente polimórficas (Fisher \& Cover 2007). Se conocen 223 especies en el mundo, doce en México y una en Jalisco (Vásquez-Bolaños 2011). Tiene una amplia distribución, aunque la mayor diversidad se concentra en ambientes áridos y semiáridos (Mackay \& Mackay 2002; Umphrey 1996).

La Sierra de Quila, es una área montañosa localizada al suroeste del estado de Jalisco, de aproximadamente 32,000 hectáreas, de las cuales 15,192 están consideradas como Área Natural Protegida (ANP). Su superficie está ubicada dentro de los municipios de Tecolotlán, Atengo, Ameca, San Martín Hidalgo y Tenamaxtlan. Con altitudes que van desde los $1300 \mathrm{msnm}$ en su zona más baja, hasta los $2560 \mathrm{msnm}$ en su punto más alto. Su clima es templado semicálido en las zonas de menor altitud 
y en las partes más altas es templado subhúmedo con lluvias en verano, los tipos de vegetación predominantes son: bosque de pino encino, bosque de encino, bosque mesófilo de montaña, bosque de galería, bosque tropical caducifolio y bosque espinoso (Guerrero-Nuño \& Lopez-Coronado 1997). La colecta se realizó en Sierra de Quila desde junio de 2007 hasta diciembre de 2010, de manera directa. Las muestras de hormigas colectadas fueron preservadas en frascos con alcohol al $85 \%$ de concentración, se etiquetaron con los datos necesarios para su incorporación a la colección. Los especímenes fueron determinados a nivel género con la ayuda de las claves de Mackay y Mackay (1989), así como Fisher y Cover (2007). Para la determinación de las especies se utilizaron las claves de Umphrey (1996) y Mackay (1989) y fueron corroboradas por el Dr. William P. Mackay de la Universidad de Texas. El material se encuentra depositado en la Colección Entomológica del Centro de Estudios en Zoología la Universidad de Guadalajara (CZUG).

Aphaenogaster punctaticeps Mackay, 1989

Tamaño medio, menor a $7 \mathrm{~mm}$. La cabeza alargada con puntuaciones en el dorso, espinas del propodeo pequeñas. Color café rojizo uniforme en todo el cuerpo (Mackay \& Mackay 2002). Se colectaron 55 individuos en diciembre 2008, marzo y octubre 2009. Todos los ejemplares fueron colectados en el suelo mediante captura directa, en bosque de pino encino con altitudes desde 1253 a $2045 \mathrm{msnm}$. En la Sierra de Quila en límites de los municipios de Tecolotlán y Tenamaxtlan. Los nidos fueron localizados debajo de piedras y bajo hojarasca, las hormigas forrajean de manera individual, los nidos son de unas cuantas decenas de obreras. La distribución conocida para esta especie es el sur de los Estados Unidos de Norteamérica: Arizona, Nuevo México y Texas (Mackay \& Mackay 2002). Esta información representa un nuevo registro para México.

\section{Aphaenogaster mexicana (Pergande, 1896)}

Longitud de $7 \mathrm{~mm}$, cuello definido, espinas propodeales cortas. Color café claro (amarillento) uniforme en todo el cuerpo (Pergande 1896). Se colectaron en el suelo, mientras forrajeaban, dos individuos, uno en septiembre 2008 y otro en julio 2009, en bosque tropical subcaducifolio, a una altitud de $1468 \mathrm{msnm}$ en la Sierra de Quila, municipio de Tecolotlán y a una altitud de $1782 \mathrm{msnm}$ en Sierra de Cacoma, municipio de Ayutla. Estas hormigas son ágiles en sus movimientos. Para México estan reportadas en los estados de: Hidalgo, Nayarit y Puebla (Vásquez-Bolaños 2011). Este es el primer registro para el Estado de Jalisco.

Con el presente trabajo se incrementa a 13 el número de especies de Aphaenogaster para México y a tres para el estado de Jalisco: Aphaenogaster ensifera Forel, 1899, A. mexicana (Pergande, 1896) y A. punctaticeps (Mackay, 1989). Cabe destacar que la distribución conocida de la mayoría de las especies es en zonas áridas o semiáridas, hacia la región Neártica, los ejemplares revisados proceden de bosques templados (Mackay \& Mackay 2002; Umphrey 1996). 
AGRADECIMIENTOS. Al Dr. William P. Mackay por la corroboración en la determinación de los ejemplares y su apoyo para el mejor conocimiento de la mirmecofauna mexicana.

\section{LITERATURA CITADA}

Fisher, B. L. \& Cover, S. P. 2007. Ants of North America. A guide to the genera. University of California Press. Los Angeles, CA.

Guerrero-Nuño, J. J. \& López-Coronado, G. A. 1997. La vegetación y la flora de la Sierra de Quila. Universidad de Guadalajara. Guadalajara, Jal.

Mackay, W. P. 1989. A new Aphaenogaster of southern New Mexico. Journal of the New York Entomological Society, 97: 47- 49.

Mackay, W. P. \& Mackay, E. E. 1989. Claves de los géneros de hormigas en México (Hymenoptera: Formicidae). Memorias del II Simposio Nacional de Insectos Sociales. Oaxtepec, Morelos, México. Sociedad Mexicana de Entomología. Pp. 1-82.

Mackay, W. P. \& Mackay, E. E. 2002. The ants of New Mexico (Hymenoptera: Formicidae). The Edwin Mellen Press. Lewinston, Ney York.

Pergande, T. 1896. Mexican Formicidae. California Academy of Sciences, 5: 558-596.

Umphrey, G. J. 1996. Morphometric discrimination among sibling species in the fulva-rudis- texana complex of the ant genus Aphaenogaster (Hymenoptera: Formicidae). Canadian Journal of Zoology, 74: 528-559.

Vásquez-Bolaños, M. 2011. Lista de especies de hormigas (Hymenoptera: Formicidae) para México. Dugesiana, 18: 95-133.

\section{Margarita VILlalVAZO-PALACIOS, ${ }^{1}$ Juan Francisco PÉREZ- DOMÍNGUEZ ${ }^{2}$ \& Miguel VÁSQUEZ-BOLAÑOS 3}

${ }^{1}$ Secretaría de Educación Jalisco, Esc. Sec. Foránea 55. Circunvalación 220. El Grullo, Jalisco. C. P. 48740.<villapalacio.mar@gmail.com>

${ }^{2}$ INIFAP- Campo Experimental Centro Altos de Jalisco Km. 8 Carr. libre Tepatitlán-Lagos de Moreno. C. P. 47600, Apartado postal 56. Tepatitlán de Morelos, Jalisco. <perez.juanfrancisco@inifap.gob.mx> ${ }^{3}$ Entomología, Centro de Estudios en Zoología, CUCBA, Universidad de Guadalajara. Km 15.5 Carr. Guadalajara-Nogales. Las Agujas, Zapopan, Jalisco. C. P.45220.<mvb14145@hotmail.com> 